\title{
Optimization Algorithm for Route Selection Based on Intelligent Optimi- zation
}

\author{
Wang Aiju ${ }^{1, *}$ and Shao $\mathrm{Hua}^{2}$

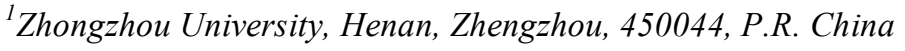 \\ ${ }^{2}$ Henan Vocational College of Chemical Technology, Henan, Zhengzhou, 450042, P.R. China
}

\begin{abstract}
This paper presents an Optimization for Route Selection based on Simulated Annealing Gene Expression Programming (ORS-SAGEP) algorithm. When optimizing multiple objective functions with the Gene Expression Programming (GEP) algorithm, the ORS-SAGEP algorithm performs simulated annealing for every individual in the initial population, and then performs a series of other genetic manipulation and fitness function evaluations. Because it is easy for the simulated annealing (SA) algorithm to jump out of local optimal solutions, to solve this problem, this paper presents the ORS-SAGEP algorithm accordingly, which can better solve single GEP's problems of poor optimization accuracy and easily falling into local optimum.
\end{abstract}

Keywords: Gene Expression Programming, Genetic Manipulation, Simulated Annealing Gene, QoS Requirements.

\section{INTRODUCTION}

As a direct carrier of Internet service, network traffic can directly reflect the network performance. A highly stable network should be capable of carrying any burst network traffic, until exceeding the maximum throughput of the network. If the network traffic exceeds the network's inherent load capacity for a long time, it is inevitable to lead to network congestion caused by excessive network traffic, and a serious decline in the overall performance of the network. Therefore, as the Internet service becomes increasingly diverse and complex, it becomes more and more important to control network traffic intelligently. However, because Internet is a complex nonlinear system, there are still a large number of questions about its dynamic characteristics that remain to be answered. Due to the complexity of network traffic, the control of it can't be as convenient as other linear and nonlinear systems. Therefore, there are still many difficulties in the research on network traffic control technology. Under most circumstances, when using optimized routing strategies, the external load may also exceed the Internet's own capacity. In such cases, if no measures taken to effectively control network traffic, the queue of the bottlenecked link will become longer, and at the same time, the delay time of network data packets will also increase to exceed the maximum delay limit for network data packet forwarding. Meanwhile, the ever-increasing length of network link queues will also deplete the buffer space on some nodes, so that network data packets arriving at these nodes will be discarded and resent, thus causing a huge waste of network resources.

*Address correspondence to this author at the School of Information Engineering, Zhongzhou University, Henan, Zhengzhou, 450044, P.R. China; Tel: 13803770071; E-mail: ywind2005@163.com
The main goal of network traffic control is to deploy reasonable congestion control mechanisms in the network for effective control of the overload traffic's flow through the network, limiting data delay and buffer overflow, finally realize efficient network operation, optimize the use of network resources, minimize or avoid Internet congestion, and improve network performance and QoS service quality.

In recent years, the research on complex network traffic control has attracted widespread attention of scholars in the field. For example, it was pointed out in Literature [1] that network traffic control plays a very important role in supporting the use of buffer space and bandwidth in various services. Effective traffic control is not only the foundation for the stable and efficient operation of the Internet, but also the foundation and precondition of various QoS service models and technologies. The network traffic control method is divided into macro layer control and micro layer control. The former focuses on the utilization ratio and operating efficiency of the entire Internet resources from an overall perspective, including such control methods as traffic engineering, and QoS routing; while the latter focuses on the control of the data stream layer, mainly including the following control methods: data packet scheduling, data packet discarding, data packet blocking, and data traffic allocation.

\section{OPTIMIZATION FOR ROUTE SELECTION BASED ON GENE EXPRESSION PROGRAMMING (ORS- GEP) ALGORITHM}

\subsection{Overview of Major Technologies}

Network traffic allocation is one of the effective means of network traffic control, while route optimization is the key technology to realize network traffic allocation. Reasonable route optimization can improve both the utilization ratio of 
network resources and the network performance. Ideal ORS (Optimization for Route Selection) strategies, reasonable network link capacity and traffic allocation can greatly facilitate the reduction of network operating costs and average delay, the improvement of network service quality, and the effective use of network resources. At present, the interior gateway protocol OSPF [2] (Open Shortest Path First) used on the Internet uses SPF (Shortest Path First) algorithm to select routes. In this algorithm, each link in the network corresponds to a manageable weight, and the SPF algorithm can pick out the path with minimum weight sum.

Route quality is directly related to network performance. In the industry, there are mainly two ways for QoS route selection optimization: control through nodes, and control through local or entire network. The former mainly optimizes through single node or single link, and uses such control strategies as service scheduling and service flow shaping to realize the service's management of the use of node shared resources (e.g., the consumption of resources such as cache, and processor); the latter realizes the direct control of service connection/service flow in the network generally through route and signaling management. At present, the difficulties in QoS route research mainly include:

(1) NP-Complete problem: This is a non-deterministic problem of polynomial complexity. When more than two independent parameters are required at the same time, it is a NP-Complete problem. For example, a multimedia video-ondemand service will request both parameters of delay and delay jitter at the same time. However, other parameters involved in the network at the same time also include jitter, bandwidth, loss rate, service cost and other considerations, and these parameters are independent. How to choose a route strategy that satisfies multiple parameter constraints is a typical NP-Complete problem [3]. The NP-Complete problem has a direct and crucial impact on the realizability of the routing algorithm.

(2) The problem of multi-service coexistence: As a network with strong "diversity and heterogeneity", while carrying many kinds of services, Internet also needs to meet different QoS requirements from different services. Therefore, how to realize the optimization of network performance and the expansion of network scale is a question that has to be thought deeply. It is difficult to determine optimal operating points, especially when best-effort is coexisting with QoS.

(3) The problem of large storage of status information: After the introduction of QoS in the system, the data volume of the status parameters that need to be recorded by nodes will increase greatly compared to that of traditional routes. If the storage of status parameters and the number of nodes in the network increase exponentially, the expansion of network scale will be limited.

(4) The problem of inaccurate information: The factors such as the jitter of the link in the transmission process and the joining/migration/exit of nodes may have some influence on the network status, and at the same time, these changes may also affect the accuracy of status information transmission and the performance of the algorithm.

At present, researchers at home and abroad has paid close attention to the network traffic allocation and ORS method.
In Literature [4], H.Pirkul and others improved the traditional Lagrangian algorithm. Experiments demonstrate that the improved algorithm can better optimize the route selection scheme. However, the problem of ORS and traffic allocation itself belongs to the problem of NP-Hard complete in the combinatorial optimization. When using a single mathematical method to work out this problem, the result is often unsatisfactory. Therefore, some researchers have proposed in recent years the use of heuristic algorithms in solving the problem of ORS. For example, in Literature [5], the author proposed a Multiple Objective Optimization based Chaotic Simulated Annealing (MOOCSA) traffic allocation method. Simulation experiments demonstrate that this method can effectively improve the utilization ratio of network resources and reduce the occurrence of network congestion, so as to improve the network performance.

In Literature [6], the author put forward a Tabu Search Algorithm (TSA) based optimization algorithm for the capacity and traffic allocation in computer communication networks. Simulation experiments demonstrate that this algorithm can effectively work out the problem of route selection and traffic allocation. Compared with traditional algorithms, the quality of solution is greatly improved, especially when compared with large-scale networks or networks under heavy loads, this algorithm is more advantageous. Ye Dazhen and $\mathrm{Wu}$ Xinyu [7] also used the Genetic Algorithm (GA) to work out the problem of network traffic allocation. After performing computer simulation toward small networks, they proved the feasibility of this algorithm.

GEP was proposed in 2001 by Candida, a Portuguese biologist [8]. GEP combines the advantages of GA and genetic programming, and uses simple coding to solve complex problems. As an efficient self-adaptive evolutionary search algorithm, GEP algorithm has been successfully applied in many fields, especially in recent years, many authors have applied GEP algorithm to the optimization of multiple objective functions. For example, in Literature [9], through designing new coding and GA, the author proposed a multiple objective optimization algorithm based on gene expression programming. Simulation experiments on standard test functions demonstrated the effectiveness of this algorithm.

In view of the advantage of the GEP algorithm in the field of function optimization, and because it is easy for the SA algorithm to jump out of local optimal solutions, this paper presents the ORS-SAGEP algorithm.

\subsection{Problem Description}

The essence of network traffic allocation is to determine the appropriate route selection by the given network topology and the actual capacity of each transmission link in the network, and then allocate corresponding bandwidth resources according to the traffic distribution of various services between the source and the destination host. Generally, the best bandwidth allocation scheme is to minimize the network delay and network congestion across the network.

When researching the problem of route selection, a network is usually expressed by a weighted graph $\mathrm{G}(\mathrm{N}, \mathrm{E})$, 


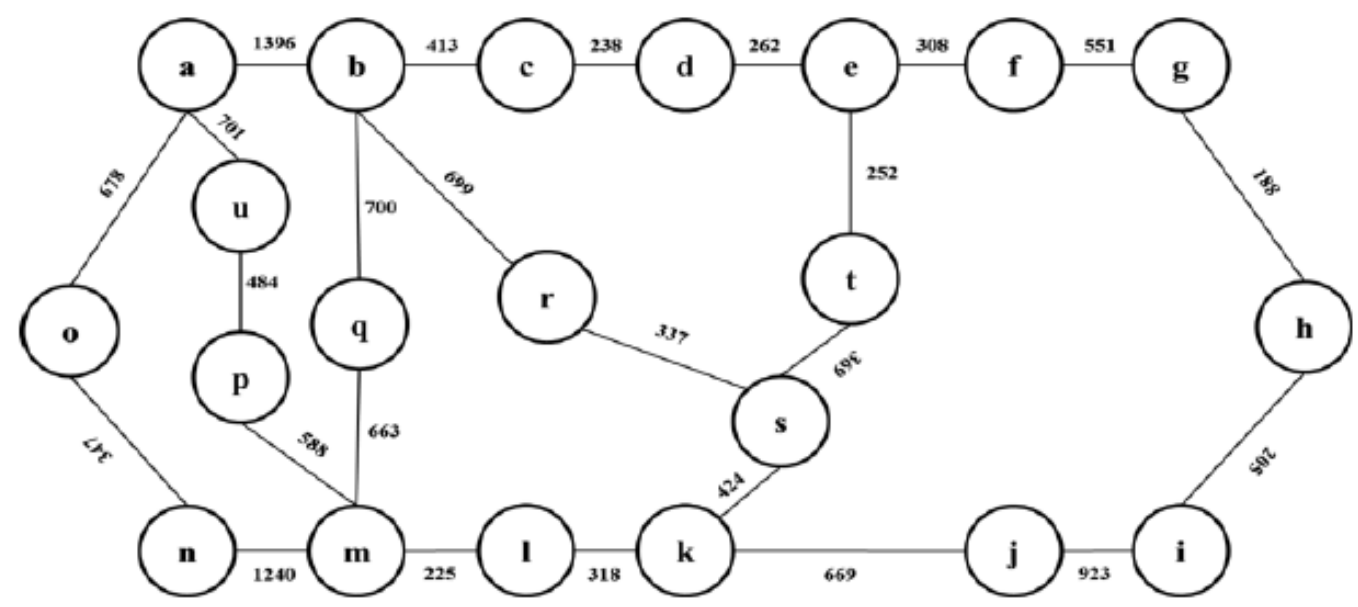

Fig.(1). ARPA Network Topology

Wherein $\mathrm{N}$ denotes the set of routing nodes, and $\mathrm{E}$ denotes the set of communication links that connect routing nodes. $|\mathrm{N}|$ and $|\mathrm{E}|$ denote the number of routing nodes and the number of links in the network respectively. ${ }^{C_{i j}}$ denotes the network transmission cost of the communication link $(i, j)$ (can be expressed as network transmission delay, and bandwidth size, etc.). When using S and D to denote the source routing node and the destination routing node respectively, and using $I_{i j}$ to denote the network connection of each link, it can be defined as follows:

$$
I_{i j}=\left\{\begin{array}{l}
1, \text { Path exists routing node } \mathrm{i} \\
\text { to the routing node } \mathrm{j} ; \\
0, \text { Path does not exist routing } \\
\text { node } \mathrm{i} \text { to the routing node } \mathrm{j} ;
\end{array}\right.
$$

Obviously, all the elements on the diagonal of ${ }^{I_{i j}}$ are 0 , and meet the conditions: If ${ }^{I_{i j}}=1$ and $I_{j k}=1$, then $I_{i k}=1$. The problem of the shortest routing path optimization can be transformed into the problem of solving the minimum value optimization, and the objective function can be expressed as:

$$
\min \sum_{i=S}^{D} \sum_{j=S}^{D} C_{i j} I_{i j}
$$

Its constraints are as follows:

$$
\sum_{\substack{i=S \\
j \neq i}}^{D} I_{i j}-\sum_{\substack{j=S \\
j \neq i}}^{D} I_{i j}=\left\{\begin{array}{c}
1, \text { if }(\mathrm{i}=\mathrm{S}) \\
-1, \text { if }(\mathrm{i}=D) \\
0, \text { otherwise }
\end{array}\right.
$$

Wherein $I_{i j} \in\{0,1\}$. Formula 2 guarantees the shortest path between the source routing node and the destination routing node, which also means the smallest network transmission delay.

\subsection{ORS-GEP Algorithm}

ORS itself is a NP problem, and in combination of the advantage of the GEP algorithm in combinatorial optimization, this paper presents the ORS-GEP algorithm.

\subsubsection{Coding}

The coding method largely determines how to perform such operations as genetic evolution of groups as well as the efficiency of these operations, so it is a critical step in the process of mining. To facilitate the description of the problem below, let's introduce a classical ARPA network topology first, as shown in Fig.(1). In this Fig, the letters from a to $\mathrm{u}$ denote nodes, while in actual network routes, they denote network routers. The values between nodes are called weights, while in actual network, they denote the time required to pass through this line, i.e. cost.

To better describe the ORS problem with the GEP algorithm, let's introduce several definitions:

Definition 1: (route optimization gene): ${ }^{G=}\left\{X_{1}, X_{2}, \ldots, X_{n}\right\}$ denote ORS genes, wherein $X_{i}, i \in(i, n)$ denotes any routing node in the network, ${ }^{X_{i}}$ denotes the route starting point, and $X_{n}$ denotes the route end point.

Definition 2: (route optimization individual): The route optimization individual is composed of one or more route optimization genes, and defined as:

$$
D=\left\{G_{1}, G_{2}, \ldots, G_{n}\right\}
$$

The number of genes in the whole route optimization individual is determined by GEP initial parameters.

Definition 3: (route selection population): The route selection population is composed of one or more route optimization individuals, and defined as:

$$
P=\left\{D_{1}, D_{2}, \ldots, D_{m}\right\}
$$

To facilitate the description, take the following as an example:

Given $R=\{a, b, c, d, e, f, g, j\}$, wherein every letter denotes one routing node, and it will be coded as a route optimization individual, as shown below:

$$
\begin{aligned}
& C_{1} \text { :achge } \\
& C_{2}: \text { cbag } \\
& C_{3} \text { :cdfag }
\end{aligned}
$$

Fig. (2) shows the spatial structure composed of the expressions of route optimization individuals $C_{1}$ and $C_{2}$, and expresses a specific route for the shortest path ORS problem wherein $\mathrm{c}$ is the route starting point, and $\mathrm{g}$ is the route end point. While ${ }^{C_{3}}$ denotes invalid route optimization individuals, because it doesn't exist in the actual routing paths. 


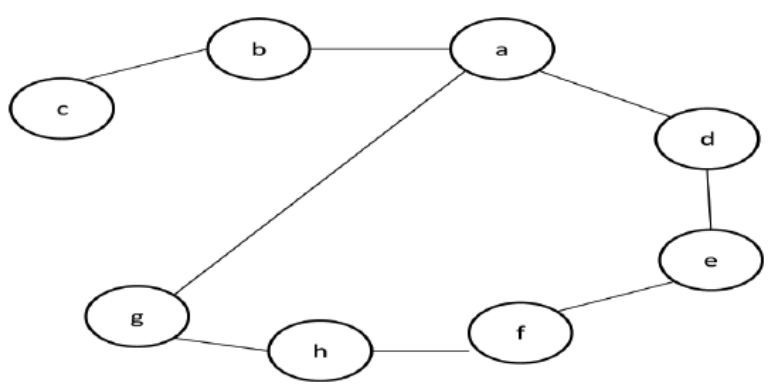

Fig.(2). Expression of Route Optimization Individual

When performing shortest path route selection with the GEP, the source and destination nodes of route optimization genes are set according to initial parameters, and at the same time, with only one identical routing node kept, so that the length of the route optimization individual designed is dynamic, which both conforms to the characteristics of route selection, and can reduce the amount of calculation when doing fitness function calculation.

\subsubsection{Genetic Manipulation}

During the process of optimization for route selection based on gene expression programming, main genetic operators include selection operators, mutation operators and crossover operators [10].

\section{(1) Selection operators}

The selection operation is to select a certain number of route optimization individuals from the route selection population through certain strategies, and meanwhile, retain them to the next generation to participate in the mutation, crossover and other genetic manipulations between route optimization individuals. The operation used in this algorithm is the commonly used roulette and best retention strategy, in which, the fitness function value of each route optimization individual in the route selection population is calculated first, and then the fitness function values are used for sorting. Generally, there are two processes: Firstly, calculation of fitness function values; secondly, sorting by the fitness function values in descending order. The route optimization individual with the largest fitness function value is unconditionally retained to the next generation, while other route optimization individuals are selected according to the roulette strategy, until the generation of the next generation population.

(2) Mutation operators

Mutation is the most important genetic way to maintain the advancement and innovation of species, and also an effective genetic manipulation capable of optimizing the global optimal solution. Based on the characteristics of route optimization genes, and to ensure that the genes obtained are valid, the entire mutation operation can only be carried out between the head and tail of the route optimization gene, and the value range of the mutation is limited to other routing nodes except the head and tail of genes.

To facilitate the description, take the following as an example: Given ${ }^{R}=\{a, b, c, d, e, f, g, j\}$, the gene head is a, the gene tail is e, the gene length is 5, a randomly generated route optimi- zation individual is $C_{1}$ :achge, and the mutation probability is 0.001 , then the mutated route optimization individual is: $C_{1}:$ adcfe.

\section{(3) Crossover operators}

In view of the characteristics of network route selection, the entire crossover operator can only use the one point crossover. To ensure the validity of the route optimization gene, the crossover operator is only between the head and tail of genes.

To facilitate the description, take the following as an example: Given $R=\{a, b, c, d, e, f, g, j\}$, the gene head is a, the gene tail is e, the gene length is 5, two randomly generated route optimization individuals are respectively ${ }^{\min \sum_{i=j}^{n} \sum_{j=s}^{n} C_{i j} I_{i j}}$, the crossover probability is 0.33 , and the crossover locations are 2 , then the two mutated route optimization individuals are respectively: $C_{1}$ :achfe, $C_{2}$ :adcge .

\subsubsection{Algorithm Description}

The entire ORS-GEP algorithm is described as follows:

\section{Algorithm 1: ORS-GEP}

Input: GEP basic parameters;

Output: BestRoute, the routing path corresponding to the optimal route optimization individual.

Begin \{

(1) $i=0$; // Initialize the evolution algebra

(2) Init(Pop); // Initialize the population

(3) Calculate the optimal route optimization individual in the current population;

(4) while $(\mathrm{i}<\mathrm{MaxGen})\{$

(5) Mutate( ); // Perform the mutation operation

(6) Select( ); // Perform the selection operation

(7) CrossOver( ); // Perform the crossover operation

(8) Calculate the optimal route optimization individual in the current population;

\section{(9) $\mathrm{i}++;\}$}

(10) The routing path set corresponding to the finally obtained optimal route optimization individuals.

$$
\text { \} }
$$

\section{ORS-SAGEP ALGORITHM}

Although the traditional single GEP algorithm has better search capability in optimizing multiple objective functions, as a member of the evolutionary algorithm family, it is also inevitably to have such defects as "precocity". To better solve the single algorithm's problem of easily falling into local optimum, and in combination of the advantage of the 
SA in local optimization, this section presents the ORSSAGEP algorithm.

The genetic manipulation itself is especially important for the global convergence of the entire GEP algorithm. In the ORS-SAGEP algorithm, the characteristics of the SA algorithm in local optimization have been fully used to avoid the utmost GEP algorithm falling into local optimum. The SA algorithm enables every individual in every generation of population of GEP to become local optimum, which can largely accelerate evolution, and make the entire algorithm run in the direction of global optimum.

\subsection{Overview of SA}

The SA algorithm [11] was proposed, by Kirkpatrick et $a l$. in IBM, based on the similarity between the physical annealing process and the general combinatorial optimization. It is an effective approximation algorithm proposed by introducing the annealing thought into the combinatorial optimization field for solving the large scale combinatorial optimization problem, especially the NP-Complete combinatorial optimization problem. It solves the combinatorial optimization problem using the Monte Carlo strategies. The SA algorithm is derived from the simulation of annealing process in thermodynamics. The algorithm is enabled to give an approximate optimal solution in the polynomial time, by slowly decreasing temperature parameters, at a given initial temperature. Annealing is similar to the "annealing" of metallurgy, while significantly different from the quenching of metallurgy. The former is a slow drop in temperature, while the latter is a rapid drop in temperature.

The simulation process of Kirkpatrick [12] algorithm is very simple, and is widely used in many real environments. Improved methods after that (such as thermal annealing, tempering annealing, chaotic annealing combined with neural networks, and other algorithms) have improved some performance of the SA algorithm from different perspectives. However, the improvement of network performance brought about by these algorithms is at the expense of time and space complexity. The monitoring nodes in network traffic monitoring models are often limited by factors such as computing power and storage space, so it's more appropriate to choose concise and practical algorithms to solve complex Internet traffic optimization problems. Based on the above considerations, this paper chooses the Kirkpatrick annealing algorithm. The basic thought and flow of the algorithm are described below:

Begin \{

(1) Initialization: the initial temperature is $T$ (should be large enough), the initial solution state is $\mathrm{S}$ (the starting point of the algorithm iteration), and the number of iteration for each initial temperature $\mathrm{T}$ is $\mathrm{L}$;

(2) for $(\mathrm{k}=1 ; \mathrm{k}<=\mathrm{L} ; \mathrm{k}++)\{$

(3) Generate a new solution S';

(4) Calculate the incremental $\Delta t^{\prime}=C\left(s^{\prime}\right)-C(s)$, of which $C(S)$ is evaluation function;

(5) if $\left(\Delta t^{\prime}>0\right)$ \{then accept $S^{\prime}$ as the new current solution $\}$; else $\{$ with the probability $\exp (t / T)$ accept $S$ ' as the new current solution;
(6) if a number of successive new solutions are not accepted, then output the current solution as the optimal solution, break;

(7) T decreases gradually, and $T \rightarrow 0$ \}

\subsection{ORS-SAGEP Algorithm}

When optimizing multiple objective functions with the GEP algorithm, the ORS-SAGEP algorithm performs simulated annealing for every individual in the initial population, and then performs a series of other genetic manipulation and fitness function evaluations. The formal description of the entire ORS-SAGEP is as shown in algorithm 2:

Input: GEP basic parameters;

Output: BestRoute, the routing path corresponding to the optimal route optimization individual.

Begin \{

(1) Init(P); // Initialize the population

(2) $\mathrm{i}=0$; // Set up the evolution algebra

(3) While (i<=MaxGen) \{

(4) Perform SA for every individual in population P, so as to produce local optimal individuals to form new populations;

(5) Mutate( ); // Perform the mutation operation

(6) Select( ); // Perform the selection operation

(7) CrossOver( ); // Perform the crossover operation

(8) Calculate and retain the optimal individual in the new population;

(9) $\mathrm{i}++$;

(10) Return BestRoute, the optimized optimal routing path.

\}

The operation of the entire algorithm is mainly controlled by the operation algebra $\mathrm{i}$ and the total number of iteration $\mathrm{N}$ of every individual in the population performing SA, so the time complexity of the entire algorithm is approximately $\mathrm{O}\left(\mathrm{i}^{*} \mathrm{~N}\right)$.

\section{EXPERIMENTAL ANALYSIS}

To verify the effectiveness of the ORS-SAGEP algorithm, simulation experiments were performed respectively in the lab environment for the ORS problems of the classical ARPA network and the randomly generated network RAN. The results of the ORS-SAGEP algorithm and those of the heuristic algorithms such as TSA, GA and SA algorithm were compared respectively. The advantages of the above algorithms were compared mainly from two aspects, namely the optimal fitness function value and the algorithm convergence performance. The whole test platform is Windows $7+$ Jdk1.5+Eclipse3.1, and all programs are implemented in the Java language.

In the ARPA network, there are 21 nodes and 26 links in total. Its topology is as shown in Fig. (1). The letter in the 
circle denotes the network node number, and the number on the link denotes the transmission delay between the two routing nodes on the link, expressed in seconds.

In the randomly generated network RAN, there are 20 nodes and 44 network links in total. Its topology is as shown in Fig. (3). The letter in the circle denotes the routing node, and the value on the link denotes the transmission delay between the two routing nodes on the link, expressed in seconds.

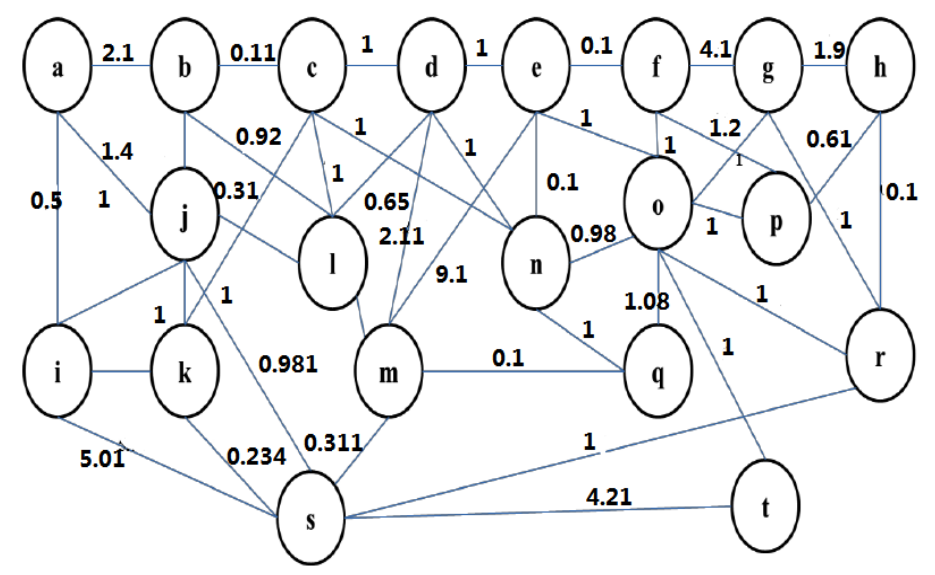

Fig. (3). RAN Network Topology

Table 1. Main Parameters of the ORS-SAGEP Algorithm

\begin{tabular}{|c|c|c|}
\hline Parameter Item & ARPA Parameter & RAN Parameter \\
\hline \hline Node Number & 21 & 20 \\
\hline Maximum Operation Algebra & 100 & 100 \\
\hline Population Size & 500 & 500 \\
\hline Selection Probability & 0.33 & 0.33 \\
\hline Mutation Probability & 0.001 & 0.001 \\
\hline Crossover Probability & 0.33 & 0.33 \\
\hline Fitness Function & $\min \sum_{i=S}^{D} \sum_{j=S}^{D} C_{i j} I_{i j}$ & $\min \sum_{i=S}^{D} \sum_{j=S}^{D} C_{i j} I_{i j}$ \\
\hline
\end{tabular}

Table 2. Shortest Path Routes Obtained by the Four Algorithms

\begin{tabular}{|c|c|c|c|c|}
\hline & $\begin{array}{c}\text { Shortest Path Route } \\
\text { of ARPA Network }\end{array}$ & $\begin{array}{c}\text { Minimum Delay } \\
\text { of ARPA Net- } \\
\text { work (s) }\end{array}$ & $\begin{array}{c}\text { Shortest Path Route } \\
\text { of RAN Network }\end{array}$ & $\begin{array}{c}\text { Minimum Delay } \\
\text { of RAN Network } \\
\text { (s) }\end{array}$ \\
\hline \hline $\begin{array}{c}\text { ORS-SAGEP } \\
\text { Algorithm }\end{array}$ & $a \rightarrow b \rightarrow q$ & 2,096 & $b \rightarrow c \rightarrow l$ & 1.11 \\
\hline TSA & $a \rightarrow b \rightarrow q$ & 2,096 & $b \rightarrow j \rightarrow l$ & 1.31 \\
\hline GA & $a \rightarrow b \rightarrow q$ & 2,096 & $b \rightarrow c \rightarrow l$ & 1.11 \\
\hline SA Algorithm & $a \rightarrow b \rightarrow q$ & 2,096 & $b \rightarrow c \rightarrow l$ & 1.11 \\
\hline
\end{tabular}


According to the ARPA network topology and the RAN network topology, the main parameters of the entire ORSSAGEP algorithm are as shown in Table 1. For the ARPA network, the start routing node of route optimization is a, and the end point is q; for the RAN network, the start routing node of route optimization is $b$, and the end point is 1 .

Experiment 1: For the ARPA network and the RAN network, run 10 times respectively using the ORS-SAGEP algorithm, TSA, GA and SA algorithm. Table 2 indicates the optimal paths and minimum delays corresponding to the shortest path routes obtained by the four algorithms. Fig. (4) compares the average delays of the above four algorithms in obtaining the optimal routing paths.

You can see from Table 2 that for the ARPA network, all of the four algorithms, namely the ORS-SAGEP algorithm, TSA, GA and SA algorithm, can work out shortest path routes: $a \rightarrow b \rightarrow q$, with a corresponding minimum delay of 2,096 seconds. For the RAN network, only the TSA in the above four algorithms fails to work out the shortest path route, with a corresponding minimum delay of 1.31 seconds; while all of the other three algorithms work out shortest path routes: $b \rightarrow c \rightarrow l$, with a corresponding minimum delay of
1.11 seconds. It also shows that the ORS-SAGEP algorithm has the same global optimization capability as traditional algorithms in working out the shortest path. For the complex network architectures of RAN, the minimum delay of the ORS-SAGEP algorithm for finding the shortest path route reduces, at most, about $18.02 \%$.

You can see from Fig. (4) and Fig. (5), for the RAN network, the minimum average delay of the ORS-SAGEP algorithm in working out the optimal routing paths is $1.13 \mathrm{sec}-$ onds, with a maximum decrease of about $3.24 \%$ compared with the average delays of the other three algorithms; for the ARPA network, the minimum average delay of the ORSSAGEP algorithm in working out the optimal routing paths is 2,096 seconds, with a maximum decrease of about $21.24 \%$ compared with the average delays of the other three algorithms. That's because the ORS-SAGEP algorithm has combined the advantage of GEP in working out combinatorial optimization and the capability of the SA algorithm in local optimization, which has greatly enhanced the global optimization capability of the ORS-SAGEP algorithm, thus ensuring that the algorithm presented in in this paper can demonstrate it's good optimization capability under the same network topology conditions.

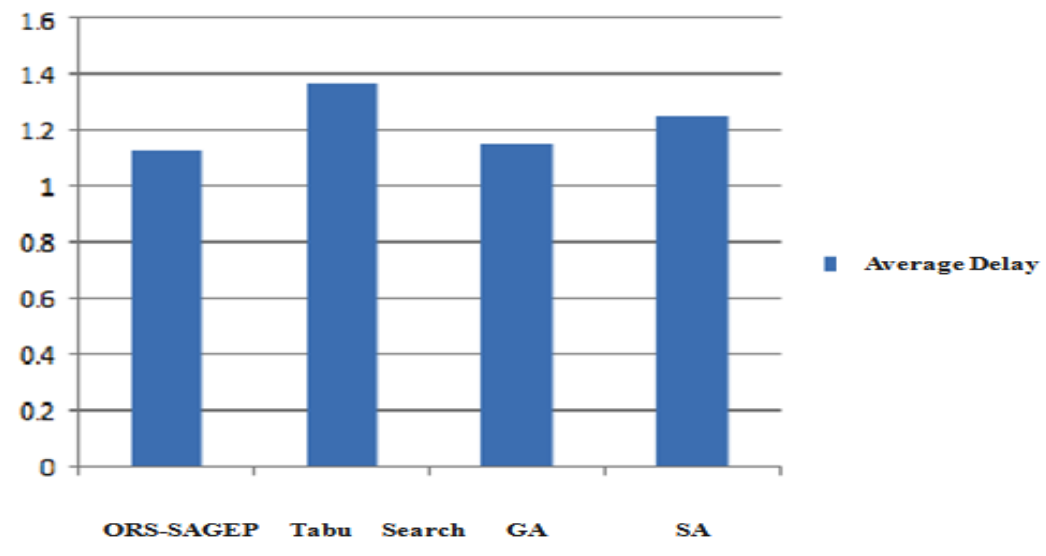

Fig. (4). Average Delay in Working out the Optimal Routing Paths of RAN.

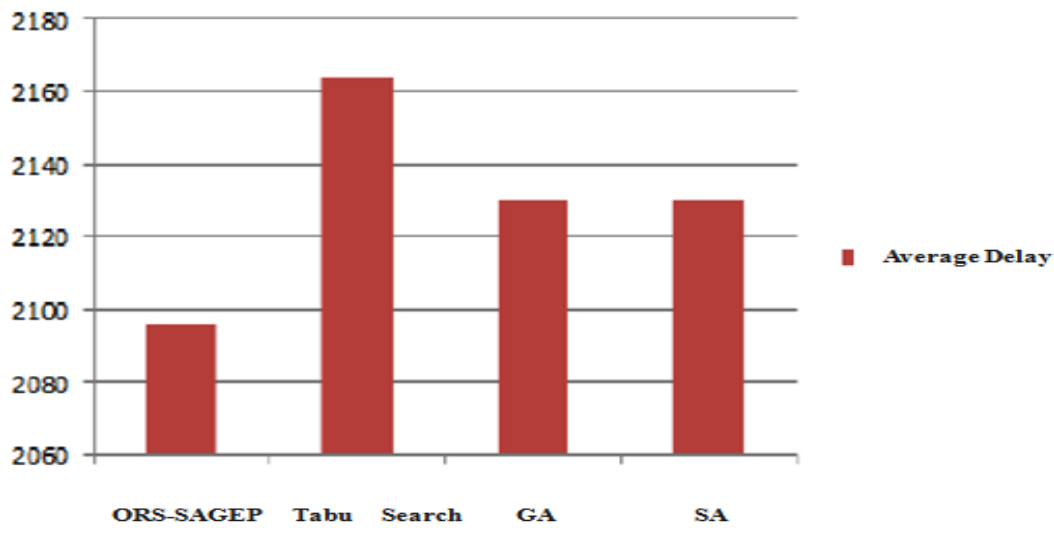

Fig. (5). Average Delay in Working out the Optimal Routing Paths of ARPA.

Experiment 2: For the ARPA network and the RAN network, run 10 times respectively using the ORS-SAGEP algorithm, TSA, GA and SA algorithm. Fig. (6) and Fig. (7) compare the times consumed by the four algorithms in work- ing out the optimal routing paths of the above two network topologies. Table $\mathbf{3}$ indicates the average convergence algebra of the above four algorithms. 


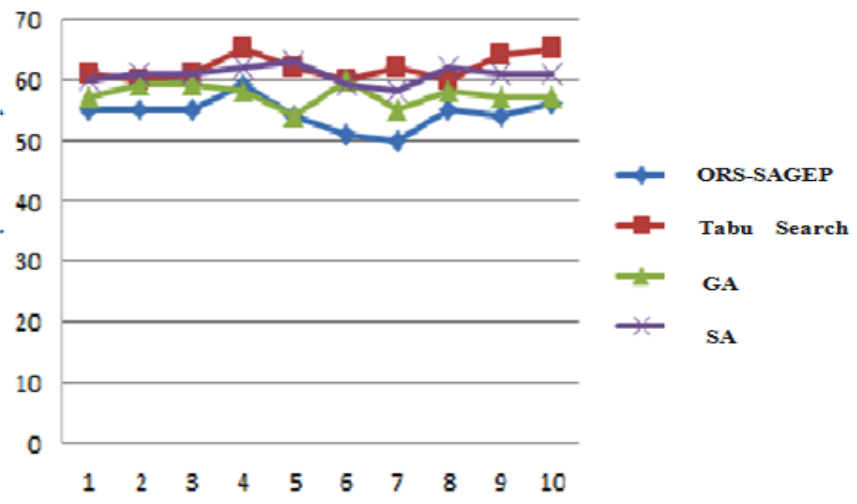

Fig. (6). Times Consumed by the Four Algorithms in Working out the Optimal Routing Paths of ARPA Network

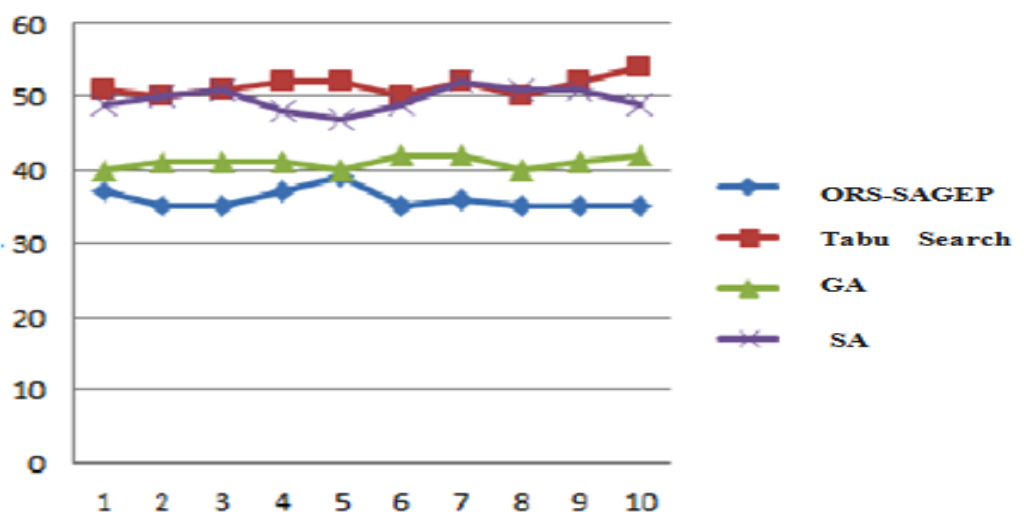

Fig. (7). Times Consumed by the Four Algorithms in Working out the Optimal Routing Paths of RAN Network.

Table 3. Average Convergence Algebra of the Above Four Algorithms.

\begin{tabular}{|c|c|c|}
\hline & $\begin{array}{c}\text { Average Convergence Algebra in Working out the } \\
\text { Shortest Path Route of the ARPA Network }\end{array}$ & $\begin{array}{c}\text { Average Convergence Algebra in Working out } \\
\text { the Shortest Path Route of the RAN Network }\end{array}$ \\
\hline \hline $\begin{array}{c}\text { ORS-SAGEP } \\
\text { Algorithm }\end{array}$ & 25 & 47 \\
\hline TSA & 44 & 30 \\
\hline GA & 30 & 35 \\
\hline SA Algorithm & 37 & 40 \\
\hline
\end{tabular}

You can see from Fig.(6) and Fig.(7), for the ARPA network, the average time consumed by the ORS-SAGEP algorithm in working out the optimal routing paths decreases by $13.97 \%$ or so, compared with those of the traditional heuristic algorithms; for the RAN network, the average time consumed by the ORS-SAGEP algorithm in working out the optimal routing paths decreases by $43.18 \%$ or so, compared with those of the traditional heuristic algorithms. This is mainly because when using the same network topologies and running algorithms for the same times, the ORS-SAGEP algorithm presented in this paper uses simple coding to solve complex problems, and meanwhile, this algorithm uses simple and practical genetic manipulation, making the algorithm fast and effective in working out the optimal solution.
Table 3 compares the average convergence algebra of the four algorithms, namely ORS-SAGEP algorithm, TSA, GA and SA algorithm, in working out the shortest path routes of the ARPA network and the RAN network. When using the same network topologies and running algorithms for the same times, the average convergence algebra of the ORSSAGEP algorithm is obviously better than other heuristic algorithms. To be specific, when working out the shortest path routes of the ARPA network, the average rate of convergence of the ORS-SAGEP algorithm increases up to 1.76 times or so; when working out the shortest routing paths of the RAN network, the average rate of convergence of the ORS-SAGEP algorithm increases up to 2.35 times or so. This is because the ORS-SAGEP algorithm combines the GEP's advantage in rapid convergence and the SA algo- 
rithm's advantage in local optimization, enabling it to find the shortest path route with less operation algebra.

\section{SUMMARY}

Route selection is an important method to realize network traffic control. The essence of network traffic control is to minimize the delay of transmitting messages in the network and reduce the network congestion as much as possible, through the optimization of route selection. Studies show that the optimization of route selection can be described through shortest routing paths. In view of the advantage of the GEP in combinatorial optimization, and in combination of the advantage of the SA algorithm in local optimization, this paper presents the ORS-SAGEP algorithm. According to the simulation results, for the ARPA network, the average delay of the ORS-SAGEP algorithm in working out the optimal routing paths decreases up to $21.24 \%$ or so, compared with traditional algorithms, and for the RAN network, the figure is $3.24 \%$ or so; for the ARPA network, the average time consumed by the ORS-SAGEP algorithm in working out the shortest routing paths decreases by $13.97 \%$ or so, and for the RAN network, the figure is $43.18 \%$ or so. When working out the shortest routing paths of the ARPA network, the average rate of convergence of the ORSSAGEP algorithm increases by about $76 \%$; when working out the shortest routing paths of the RAN network, the average rate of convergence of the ORS-SAGEP algorithm increases by about $57.5 \%$.

\section{CONFLICT OF INTEREST}

The author confirms that this article content has no conflict of interest.

\section{ACKNOWLEDGEMENTS}

Declared none.

\section{REFERENCES}

[1] Browning, Douglas. "Flow control in high-speed communication networks". Communications. vol. 5, pp. 2480-2489, 2004.

[2] Fortz, B.; Thorup, M. "Internet traffic engineering by optimizing OSPF weights". Nineteenth Annual Joint Conference of the IEEE Computer and Communications Societies. Proceedings. IEEE. Kuala Lumpur, 2000.

[3] Beard, R.A.; Lamont, G.B." Determination of Algorithm Parallelism in NP-Complete Problems for Distributed Architectures". Distributed Memory Computing Conference, USA, 1990.

[4] Sridhar Nararsimhan, Hasan Pirkul. Route selection in backbone data communication Networks. Computer Networks and ISDN Systems, vol. 15, pp. 121-133, 1988.

[5] Liu RunJie, Shen Jinyuan, Mu Weixin. "Research Communication Network Traffic Allocation Method". First class Chinese university communications departments Symposium, USA, 2007.

[6] Shen Jian, Xu Fuyong. "Optimization of rhyme and flow capacity allocation based on tabu search algorithm in computer communication networks". Journal of Lanzhou University, vol. 39, pp. 35-39, 2003.

[7] Ye Dazhen, Wu Xinyu. "Computer communication network routing and capacity allocation genetic algorithm". Journal of Electronics, vol. 24, pp. 75-78, 1996.

[8] C. Ferreira. "Gene Expression Programming: A New Adaptive Algorithm for solving Problems". Complex Systems, vol. 13, pp. 87-129, 2001.

[9] Xiang Yong, TANG Changjie, Zeng Tao, Liu Yintian, Qiao ShaoJie. "Multi-objective optimization based on gene expression programming algorithm", Journal of Sichuan University (Engineering Science Edition), vol. 39, pp. 124-129, 2007.

[10] C. FERREIRA. Gene Expression Programming: Mathematical Modeling by an Artificial Intelligence ( $2^{\text {nd }}$ Edition $), 2006$, Springer, pp. 110-130.

[11] Keikha, M.M. "Improved Simulated Annealing Using Momentum Terms. Intelligent Systems", Modelling and Simulation (ISMS), 2011 Second International Conference. 2011.

[12] Tielin Li; Yamei Yang; Zhibin Liu. “An Improved Neural Network Algorithm and Its Application on Enterprise Strategic Management Performance Measurement Based on Kirkpatrick Model" Intelligent Information Technology Application, 2008. IITA '08. Second International Symposium. USA, 2008.

Received: December 15, 2014

Revised: January 04, 2015

Accepted: February 25, 2015

(C) Aiju and Hua; Licensee Bentham Open.

This is an open access article licensed under the terms of the Creative Commons Attribution Non-Commercial License (http://creativecommons.org/licenses/by$\mathrm{nc} / 4.0 /$ ) which permits unrestricted, non-commercial use, distribution and reproduction in any medium, provided the work is properly cited. 